Errata

\title{
Comments on Pressure-Strain Correlation Models
}

By Ronald M.C. So and Richard L. Peskin. Z. angew. Math. Phys. 31, 56-65 (1980)

We would like to express our thanks to Professor S. Masuda of Keio University, Yokohama, Japan, for bringing to our attention the numerous printing mistakes in our paper and the algebraic error we made in deriving equation (18).

In view of this, the following corrections should be noted:

1. A closing $\}$ and a $\rho$ are missing in equation (1) on page 57.

2. A negative sign in front of the term $\left(\hat{C}_{1} / T\right)\left(\overline{u_{3}^{2}}-q^{2} / 3\right)$ is missing in equation (13) on page 59 .

3 . The sign in front of $2 S_{R}$ in equation (15) should be positive while the sign in front of $2 S_{R}$ in equation (17) should be negative.

4. Equation (18) on page 60 should read:

$$
-\bar{\tau}=\frac{\lambda^{2} B^{3 / 2}}{b_{1}^{3}}\left[1-\frac{4\left(1-2 \gamma_{1}\right)}{B} R i-\frac{8 \gamma_{1}}{B} S_{R}^{2}\right]^{3 / 2} \Gamma^{2}
$$

and the expression for B should read

$$
B=\frac{b_{1}}{3}-a_{0} b_{1}-\frac{2}{3}+4 \gamma_{1}\left(1-\gamma_{1}\right)-\frac{4}{3} a_{1}\left(1-a_{1}\right)
$$

5. Equation (19) on page 61 should read:

$$
1-\frac{4\left(1-2 \gamma_{1}\right)}{B} R i_{c r}=0
$$

6. The term $\overline{u_{3}^{2}} / \overline{u_{2}^{2}}=0.37$ in the second line from the bottom of page 61 should read

$$
\overline{u_{3}^{2}} / u_{1}^{2}=0.37
$$

7. The values of the constants in Table 1 under the item 'Present' on page 62 should read

$\begin{array}{llll}a_{0} & a_{1} & \gamma_{1} & C_{1} \\ 0.002 & 0.19 & -0.05 & 2.05\end{array}$

8. In Section 4 on page 62 , the second line under the section heading should read: "the rapid distortion terms in (10) ...'

9. All $\hat{b}_{1}$ 's in Section 4 should be replaced by $b_{1}$.

The corrections, as noted above, by no means change the conclusions drawn in the paper. The conclusions are essentially correct as stated. 